\title{
FIRST RUN 2 SEARCHES FOR EXOTICA AT CMS*
}

\author{
Suzan BaşeĞMez du Pree \\ on behalf of the CMS Collaboration \\ Centre for Cosmology, Particle Physics and Phenomenology (CP3) \\ Université Catholique de Louvain, Belgium \\ suzan.basegmez@cern.ch \\ (Received May 13, 2016)

\begin{abstract}
An overview of the first results of the experimental searches for exotica at the CMS experiment with $13 \mathrm{TeV}$ collision data is presented. The results cover various models with different topologies such as searches for new heavy resonances, extra space dimensions, black holes and dark matter. The analysis results with $13 \mathrm{TeV}$ data are emphasized, corresponding to an integrated luminosity in the range of $2.1-2.8 \mathrm{fb}^{-1}$, and the results are presented in comparison to those obtained with $8 \mathrm{TeV}$ data.
\end{abstract}

DOI:10.5506/APhysPolB.47.1379

\section{Introduction}

High-energy collider experiments are great opportunities to test our understanding of nature and its fundamental interactions. Many of the predictions by the Standard Model (SM) have been verified by experimental measurements with high precision. In particular, the discovery of an SM-like Higgs boson, the key element to explain the particle masses, has already been reported by the CMS and ATLAS collaborations [1, 2].

There are still many open questions awaiting to be answered. For example: why is there a matter-antimatter asymmetry in the Universe? What is the dark matter and dark energy? Is unification of the forces possible? Moreover, how can gravity fit into this model?

Many beyond the SM theories attempt to answer these questions. With the collected data at the center-of-mass energies at $8 \mathrm{TeV}$ and $13 \mathrm{TeV}$ in proton-proton collisions at the LHC, it is possible to test many different theoretical models predicting new phenomena. Exotica searches at the CMS

* Presented at the Cracow Epiphany Conference on the Physics in LHC Run 2, Kraków, Poland, January 7-9, 2016. 
experiment [3] are performed in different final states, not only to test the predictions of theoretical models but also to discover new phenomena which might be surprisingly unexpected after all.

The results presented in this paper are mainly the recent preliminary results performed with up to $2.8 \mathrm{fb}^{-1}$ proton-proton collision data collected at the center-of-mass energy of $13 \mathrm{TeV}$ at the LHC Run 2. Furthermore, the results of the similar analyses obtained with the collision data of $20 \mathrm{fb}^{-1}$ at $8 \mathrm{TeV}$ center-of-mass-energy during the LHC Run 1 data taking are shown as well. Exotica analysis results presented in this paper are mainly focused on new heavy resonance searches with different final states, as well as dark matter and black hole searches. A complete collection of all analysis results can be found in references [4] and [5].

\section{Searches for new phenomena with dijet events}

\subsection{Searches for dijet resonances}

Searches for narrow resonances with dijet events are performed in a model-independent way using the invariant mass distribution of two leading jets in quark-quark, quark-gluon and gluon-gluon final states. The integrated luminosity used for the analysis corresponds to $2.4 \mathrm{fb}^{-1}[6]$.
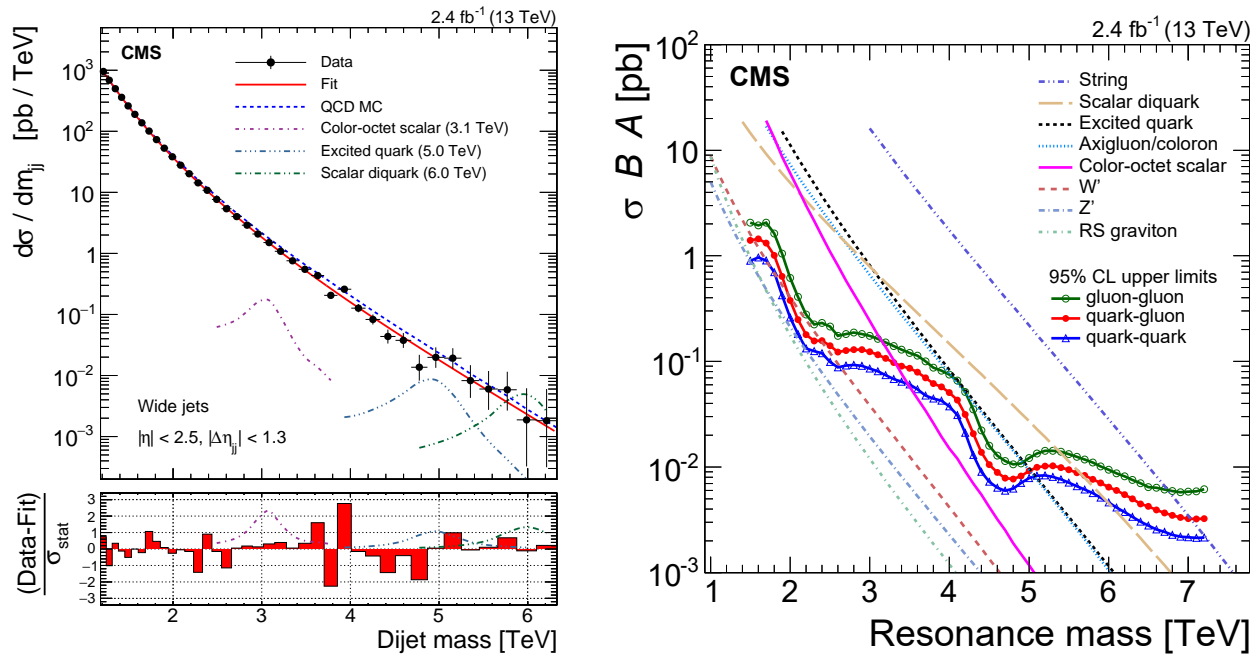

Fig. 1. Left: The invariant mass spectrum of dijet events are compared to parametrized background prediction (solid curve) as well as the QCD MC prediction (dashed line). Right: Observed and expected upper limits at 95\% C.L. are shown with various benchmark model scenarios. 
The obtained dijet invariant mass distribution is shown in Fig. 1 (left). The highest observed dijet event mass is around 6.1 TeV. The obtained spectrum is compared to leading-order Monte Carlo (MC) predictions with PYTHIA 8 as well as a parametrized background fit.

No evidence for a narrow resonance is observed and upper limits at $95 \%$ confidence level (C.L.) are set on the production cross section for narrow resonances with masses above $1.5 \mathrm{TeV}$, which is shown in Fig. 1 (right). The benchmark models considered for the limit-setting procedure are s-channel dijet resonances: string resonances, scalar diquarks, axigluons, colorons, exited quarks $\left(q^{*}\right)$, color octet scalars, new gauge bosons like $W^{\prime}, Z^{\prime}$ and Randall-Sundrum (RS) gravitons [6]. The obtained lower mass limits are already significantly better than previously published limits with Run 1 data [7].

\subsection{Searches with the dijet angular distribution}

Quark compositeness and extra spatial dimensions are tested using the angular distributions of dijet events with respect to the beam axis, defined as $\chi_{\text {dijets }}=e^{\left|y_{1}-y_{2}\right|}$, where $y_{1}$ and $y_{2}$ are the rapidities of the two jets [8]. The $\chi_{\text {dijets }}$ distribution is similar for underlying processes of quark-quark, quark-gluon or gluon-gluon interactions and is nearly flat. Any deviation in the distribution from flatteness, mainly towards low values of $\chi_{\text {dijets }}$, can give a hint for new signatures.

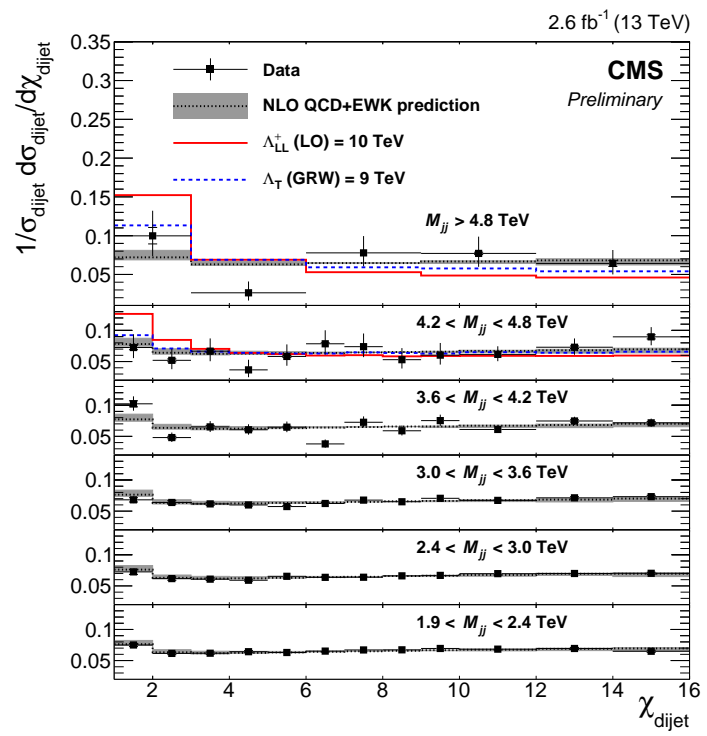

Fig. 2. (Color online) Normalized $\chi_{\text {dijets }}$ distribution for collision events collected at Run 2. Non-perturbative QCD predictions (dotted black line) and two signal predictions for QCD + contact interaction at $10 \mathrm{TeV}$ (solid red line) and QCD $+\mathrm{ADD}$ with $\Lambda_{T}$ at $9 \mathrm{TeV}$ (dashed blue line) are shown as well. 
Figure 2 shows the normalized distribution of $\chi_{\text {dijets }}$ for an integrated luminosity of $2.6 \mathrm{fb}^{-1}$, where each bin is compared to the QCD predictions including electroweak corrections. No significant deviations from the predictions are observed and upper limits for various contact interactions and ADD models are set. The $95 \%$ lower limits for the contact interaction scale $\Lambda$ are in the range of $12.1-16.3 \mathrm{TeV}$, and the lower limits for the scales of other extra space dimension models are in the range of $7.7-10.8 \mathrm{TeV}$. The obtained lower scale limits for ADD models are the most stringent limits up to date.

\section{Searches for $Z^{\prime}$}

\subsection{Searches with dileptons}

Various models beyond the SM predict the existence of spin-1 neutral gauge bosons decaying to an electron-positron or a muon-antimuon pair. Generic searches for narrow dilepton (dielectron or dimuon) resonances are usually referred to as searches for $Z^{\prime}$ resonances. A benchmark model commonly used for searches is called the Sequential Standard Model (SSM) in which the couplings of new resonance to the fermions are assumed to be identical to those of $Z$.

The searches are performed using the invariant mass distribution of dileptons for about $2.8 \mathrm{fb}^{-1}$ of Run 2 [9] data, similar to analyses performed with Run 1 data [10]. The invariant mass spectra obtained for electron and muon pairs are shown in Fig. 3 and are compared to the expected background spectra which are mainly dominated by the Drell-Yan $(Z / \gamma *)$ production of lepton pairs.
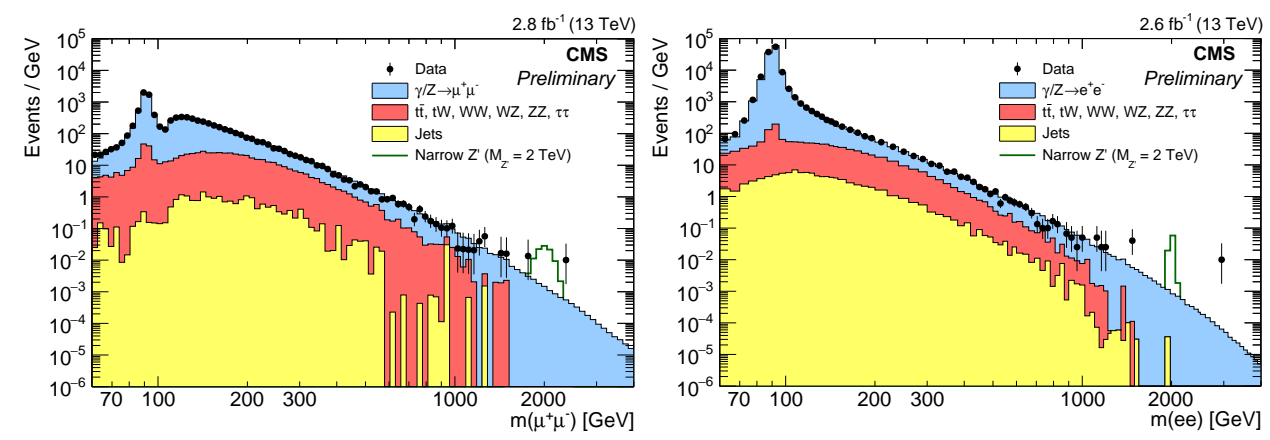

Fig. 3. The dilepton invariant mass spectra for $13 \mathrm{TeV}$ collision data. Left: $2.8 \mathrm{fb}^{-1}$ of collected dimuon events. Right: $2.6 \mathrm{fb}^{-1}$ of collected dielectron events.

The obtained spectra are smooth and the highest mass value is obtained for an electron pair with invariant mass of $2.9 \mathrm{TeV}$. The upper limits, at $95 \%$ C.L., are set to the ratio of the $Z^{\prime}$ cross section to the $Z / \gamma *$ cross 
section in a mass window of 60 to $120 \mathrm{GeV}$. The limit setting procedure is identical to the Run 1 analysis [10]; combined limit results are shown in Fig. 4 for Run 1 at $8 \mathrm{TeV}$ and Run 2 at $13 \mathrm{TeV}$ collision data. The lower mass limits set on a signal model of the SSM $Z^{\prime}$ with Run 2 data is $3.15 \mathrm{TeV}$ and surpasses the previously published result of $2.9 \mathrm{TeV}$ obtained with $8 \mathrm{TeV}$ data [10].
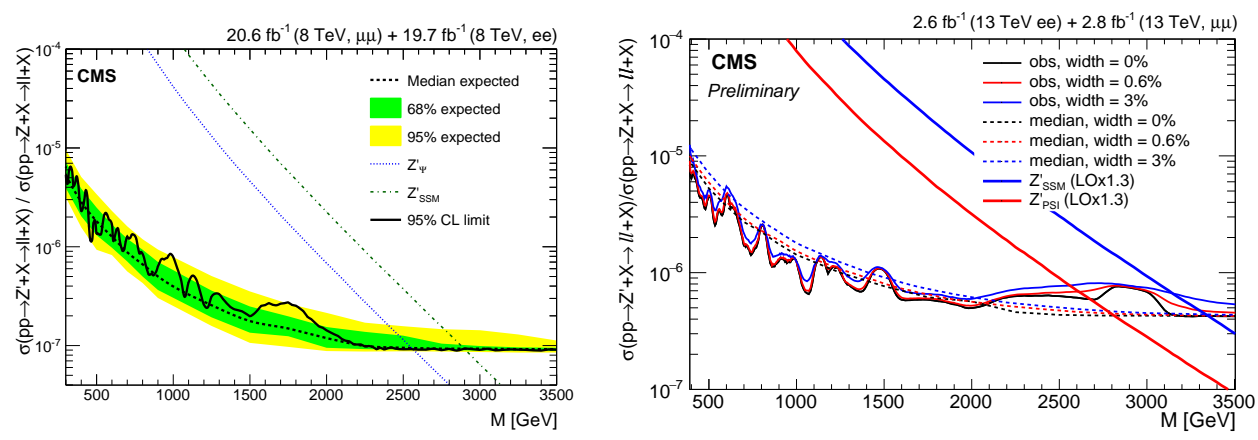

Fig. 4. The observed and expected limits set using combined dielectron and dimuon events for (left) $8 \mathrm{TeV}$ and (right) $13 \mathrm{TeV}$. The expected cross sections of two benchmark models for SSM $Z^{\prime}$ and $Z_{\psi}^{\prime}$ are shown as well.

\subsection{Searches with a Matrix Element Method}

In order to increase the sensitivity of the searches, the use of a Matrix Element Method is investigated. In principle, this method is capable of exploiting the full kinematic information of all measured particles contained in the observed events [11]. A dedicated program MadWeight [12], based on MadGraph [13], has implemented this method and is used to generate the corresponding "weights" per observed event. The analysis is performed with normalized weights combined in a likelihood.

Results of validation studies are shown in Fig. 5 for background-only and signal plus background scenarios. For the SSM $Z^{\prime}$ signal mass of $1 \mathrm{TeV}$, the local significance obtained with the Matrix Element Method is about $10 \%$ better than the results obtained using the dimuon invariant mass distribution, for more information about the studies, see Ref. [11].

This study allows for possible improvements in the searches for new resonances with dimuon events. In case a significant excess is seen in the invariant mass distribution of dimuon events, the Matrix Element Method can be used to determine the model parameters that describe best the observed data. 

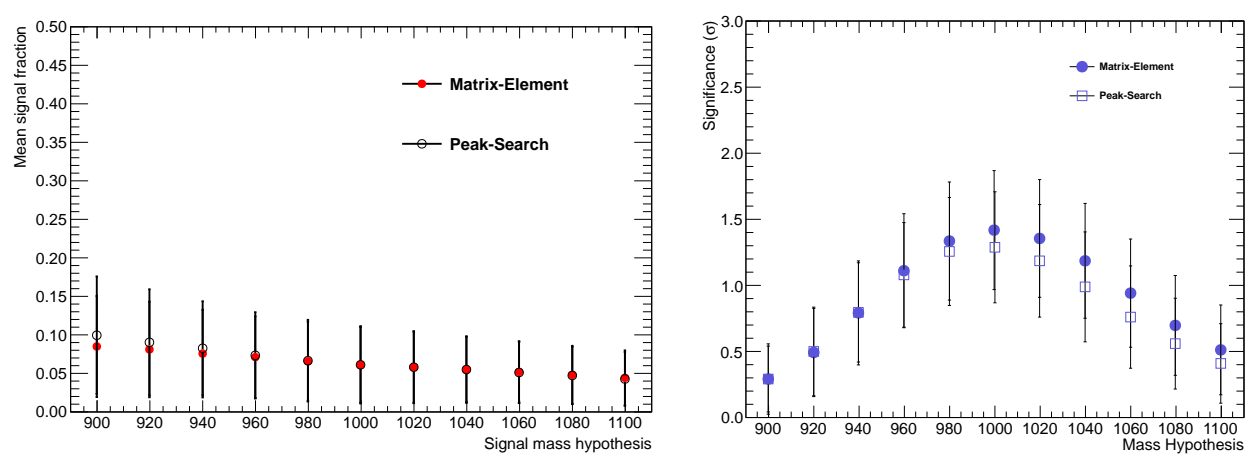

Fig. 5. Validation results obtained using the invariant mass distribution of dimuon events and the Matrix Element Method. Left: Mean estimated signal fraction with respect to hypothesized signal mass points used in the signal model with toy-MC experiments of 20 background-only events. Right: The mean significance ( $z$-score) with respect to the hypothesized signal mass points using toy-MC experiments of 20 background and 5 signal events.

\section{Search for $W^{\prime}$ \\ 4.1. Search for $W^{\prime}$ with lepton $+M E T$}

Various theories attempt to extend the SM with additional heavy gauge bosons, like a $W^{\prime}$ decaying to a charged lepton (electron or muon) and a neutrino. If the $W^{\prime}$ is sufficiently massive, the decays to a top and antibottom quark are also possible. The search for such extra bosons has been performed with $2.2 \mathrm{fb}^{-1}$ data at $13 \mathrm{TeV}$ [14]. The main discriminant variable used is the transverse invariant mass, $M_{\mathrm{T}}$, which is defined as $M_{\mathrm{T}}=\sqrt{2 p_{\mathrm{T}}^{l} E_{\mathrm{T}}^{\text {miss }}\left(1-\cos \left(\Delta \phi\left(\vec{p}_{\mathrm{T}}^{l}, \vec{p}_{\mathrm{T}}^{\text {miss }}\right)\right)\right)}$. Here, $\vec{p}_{\mathrm{T}}^{\text {miss }}$ is the negative sum of the transverse momenta of all the reconstructed particles, $p_{\mathrm{T}}^{l}$ is the reconstructed lepton transverse momentum and $\Delta \phi$ is the difference in azimuthal angles between the lepton $\vec{p}_{\mathrm{T}}$ and $\vec{p}_{\mathrm{T}}^{\text {miss }}$.

Figure 6 shows the $M_{\mathrm{T}}$ distributions for two channels, $e+E_{\mathrm{T}}^{\mathrm{miss}}$ and $\mu+E_{\mathrm{T}}^{\text {miss }}$, and the signal distributions corresponding to $W^{\prime}$ masses of 2.4 and $3.6 \mathrm{TeV}$. The events with highest $M_{\mathrm{T}}$ are around $2.0 \mathrm{TeV}$ for the electron channel and $1.3 \mathrm{TeV}$ for the muon channel. No significant deviations from the SM background predictions are observed and upper limits are set, which are shown in Fig. 7 by combining the electron and muon channels. The mass limit extends to $4.4 \mathrm{TeV}$, which improves significantly the results obtained with the $8 \mathrm{TeV}$ data [15]. 

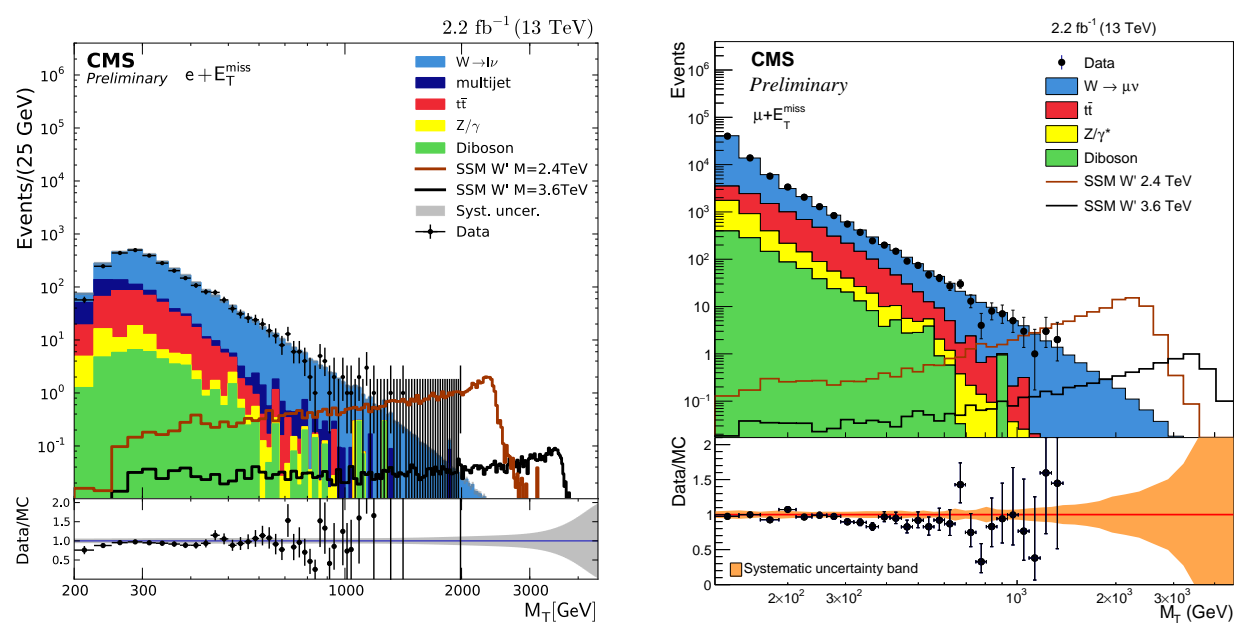

Fig. 6. The invariant transverse mass $M_{\mathrm{T}}$ distribution in (left) the $e+E_{\mathrm{T}}^{\mathrm{miss}}$ and (right) the $\mu+E_{\mathrm{T}}^{\mathrm{miss}}$ decay channel.
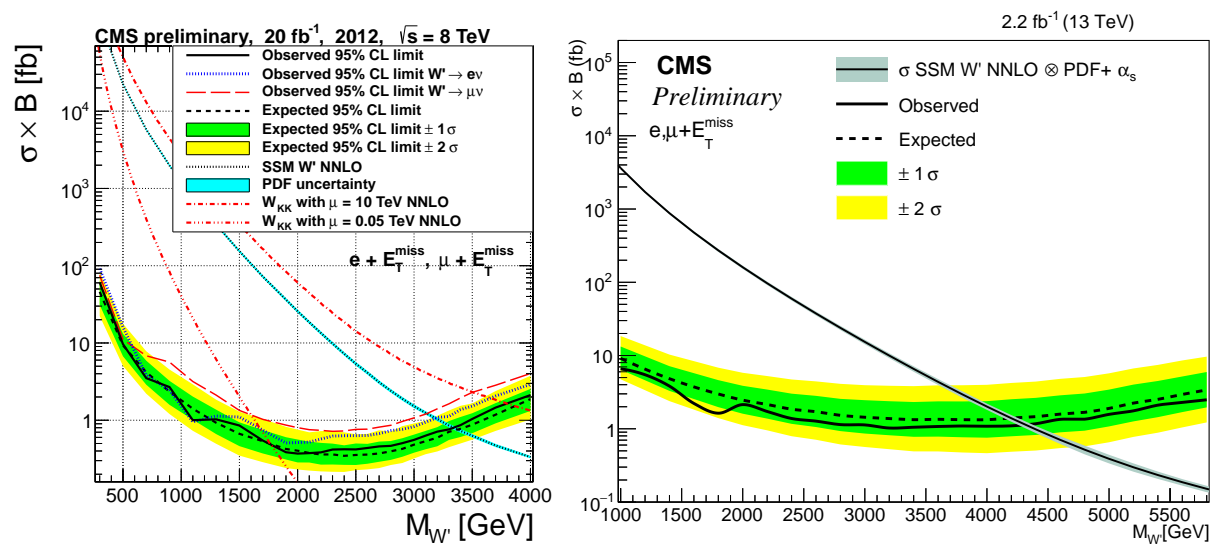

Fig. 7. The observed and expected upper limits obtained by combining $e+E_{\mathrm{T}}^{\mathrm{miss}}$ and $\mu+E_{\mathrm{T}}^{\text {miss }}$ channels (left) with $8 \mathrm{TeV}$ and (right) with $13 \mathrm{TeV}$ collision data.

\subsection{Search for $W^{\prime}$ with $t+b$}

In some of the beyond SM scenarios, a $W^{\prime}$ can strongly couple to thirdgeneration quarks. Searches for a right-handed $W^{\prime} \rightarrow t b$ are performed by considering the top-quark leptonic decays $t \rightarrow b W \rightarrow b l \nu$ with $2.2 \mathrm{fb}^{-1}$ integrated luminosity at $13 \mathrm{TeV}$ [16]. The collected events are split into categories depending on the number of $b$-tagged jets (one or two) and leptons identified as electrons or muons. The invariant mass distribution for $t b$ 
reconstructed events are shown in Fig. 8 for electrons and muons with two $b$-tagged jets. Upper limits, obtained by combining the lepton channels and the number of $b$-tagged jet event categories, are shown in Fig. 9. Despite the fact that the integrated luminosity used in the analysis is an order of magnitude smaller, the obtained limits are already better than the ones obtained with the $8 \mathrm{TeV}$ data [17].
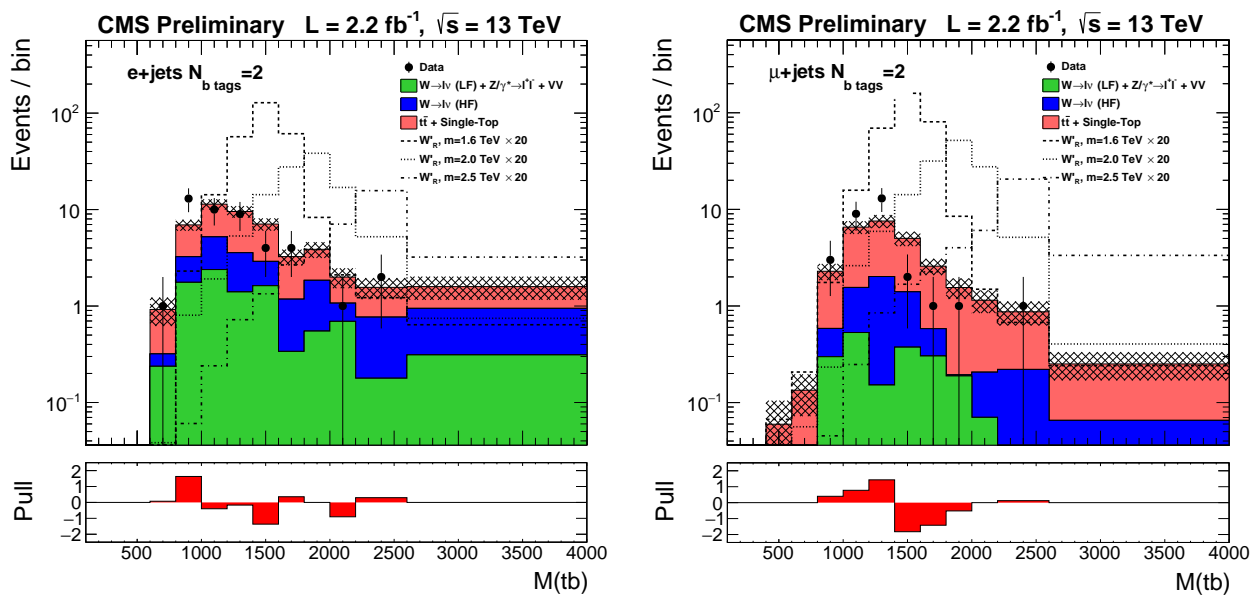

Fig. 8. The invariant mass distribution for $t b$ events with two $b$-tagged jets and leptons identified as (left) electrons and (right) muons.

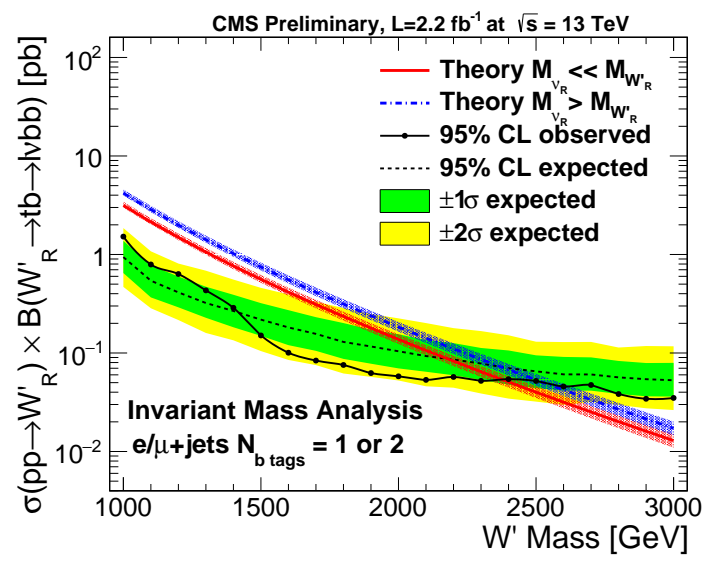

Fig. 9. Expected and observed upper limits at 95\% C.L. for the combination of all channels. 


\section{Search for Black Holes}

Semi-classical or quantum Black Holes (BHs) are searched at CMS in the high-multiplicity events [18]. The analysis with Run 2 data is performed similar to the Run 1 analysis, where the discriminant variable is defined as the scalar sum of the transverse energies of all the reconstructed particles in the event as

$$
S_{\mathrm{T}}=\left(\sum_{i=1}^{N} E_{\mathrm{T}, i}\right)+\left(E_{\mathrm{T}}^{\text {miss }}>50 \mathrm{GeV}\right) .
$$

The scalar sum of the reconstructed number of objects $(N)$ consists of the energies of jets, electrons, muons, photons and the missing transverse energy if greater than $50 \mathrm{GeV}$. The main challenge of the analysis is to

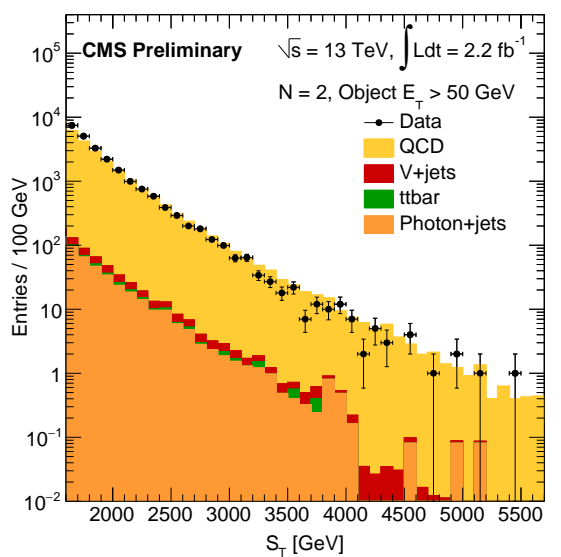

$2.2 \mathrm{fb}^{-1}(13 \mathrm{TeV})$

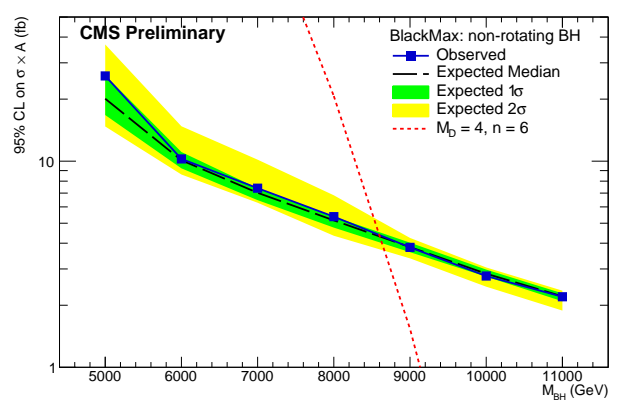

$2.2 \mathrm{fb}^{-1}(13 \mathrm{TeV})$
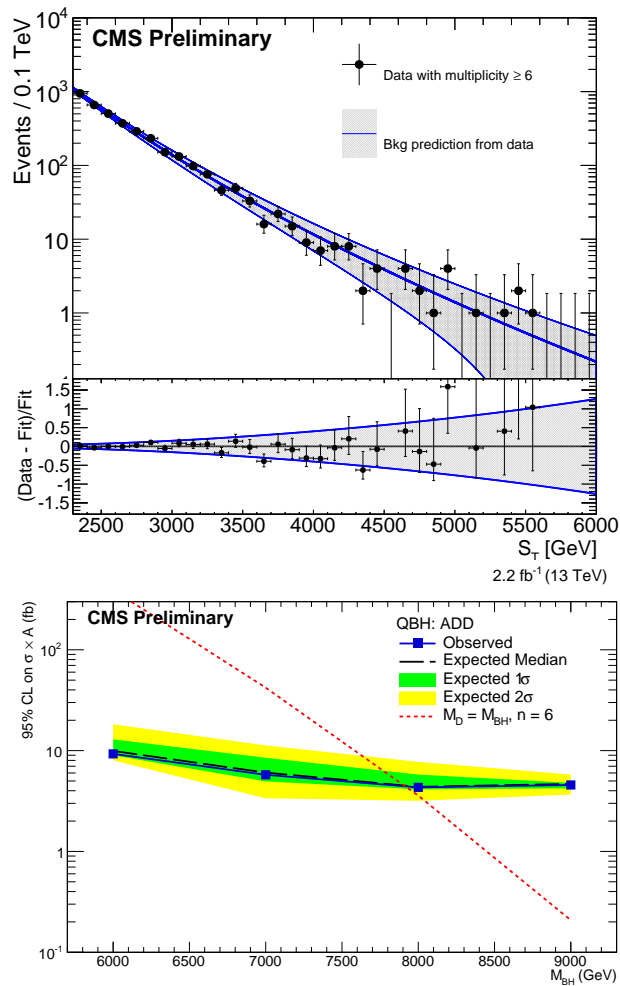

Fig. 10. Top left: The $S_{\mathrm{T}}$ distributions for data and the predicted backgrounds for $N=2$. Top right: The distribution for $N \geq 6$, where the predicted background shape is obtained with data-driven methods. The observed and expected upper limits are shown (bottom left) for semi-classical and (bottom right) for quantum BHs. 
describe the large QCD multijet background, which is difficult to predict by MC simulations. Thus, the irreducible multijet background is estimated via data-driven methods directly from the collision data, assuming the shapes of the $S_{\mathrm{T}}$ distributions are the same for low-multiplicity and high-multiplicity object categorizations above a certain threshold. By taking the shape of the dijet events for low $S_{\mathrm{T}}$, the distributions for higher multiplicities are normalized to low $S_{\mathrm{T}}$ ends, where the signal contamination is predicted to be negligible. The searches are performed with the binned $S_{\mathrm{T}}$ distributions with respect to $N$ using $2.2 \mathrm{fb}^{-1}$ of Run 2 data.

Figure 10 shows the $S_{\mathrm{T}}$ distribution for $N=2$ and the same distribution for $N \geq 6$ with the predicted background shape as well as the observed and expected upper limits, for semi-classical and quantum BHs with respect to the $\mathrm{BH}$ mass, which are translated into specific $\mathrm{BH}$ models. The minimum $\mathrm{BH}$ mass obtained for semi-classical and for quantum BHs with the first Run 2 analysis are around $8.7 \mathrm{TeV}$ and $8 \mathrm{TeV}$, respectively, which improves the previous lower limits 5.5-6 TeV obtained with the Run 1 data [19].

\section{Search for dark matter}

Searches for dark matter (DM) in Run 2 are performed using events with large missing energy and at least one jet with large transverse momentum, with the collected data of $2.1 \mathrm{fb}^{-1}$ [20]. Following the recommendations from the ATLAS and CMS DM forum [21], the interpretations in Run 1 analyses are realized in terms of simplified models, where the signal models are the mediators considering a spin-1 (vector or axial-vector) or a spin-0 (scalar or pseudoscalar) interactions between the SM and DM particles [22, 23].

The analysis is performed by a fit to the observed $E_{\mathrm{T}}^{\text {miss }}$ distribution and the results of the search are interpreted in terms of vector mediators where DM is produced in a pair. The observed data in Run 2 analysis is found to agree with the SM predictions and the limits on vector mediator are set in the plane of DM masses $\left(m_{\mathrm{DM}}\right)$ versus mediator masses $\left(m_{\mathrm{MED}}\right)$ which is shown in Fig. 11 (left) in comparison to the results by various other DM searches and Run 1.

The interpretation of results in terms of simplified models allows for a comparison to direct detection experiments as well as the cosmological relic density by WMAP and Planck experiments [24, 25]. In Fig. 11 (right), the limits are shown in terms of $m_{\mathrm{DM}^{-}}-\sigma_{\mathrm{SI}}$, where $\sigma_{\mathrm{SI}}$ is the spin-independent DM-nucleon scattering cross section. The CMS analysis is complementary to direct-detection experiments, contributing particularly to the sensitivity at low DM masses. The Run 2 analysis excludes the vector mediator masses up to $1.3 \mathrm{TeV}$, at $90 \%$ C.L., which is almost as sensitive as the Run 1 analysis, compared to the Run 1 mass limits up to $1.6 \mathrm{TeV}$. 

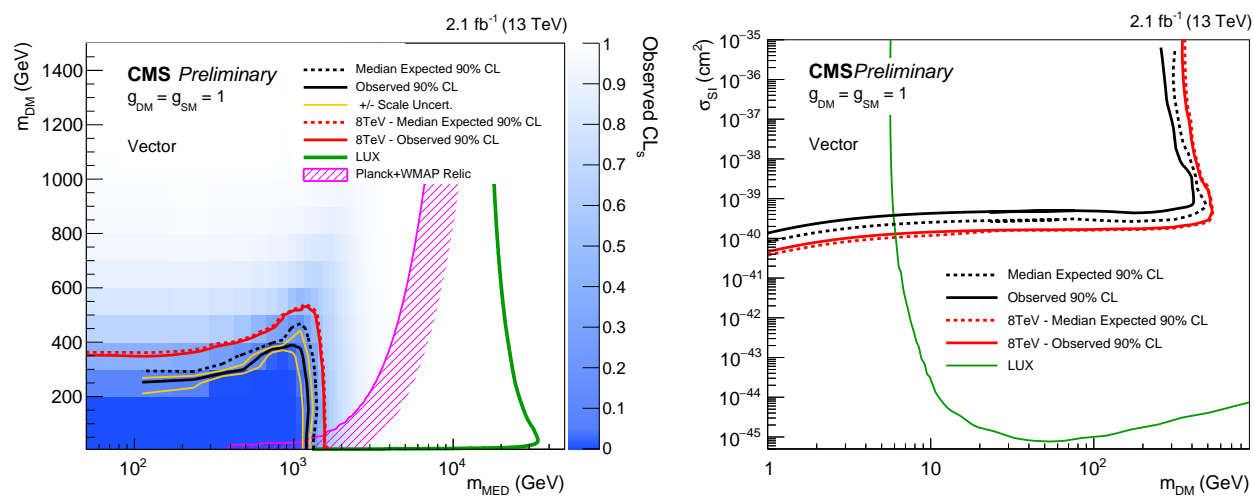

Fig. 11. Exclusion contours (left) in the $m_{\mathrm{MED}}-m_{\mathrm{DM}}$ plane in comparison to the direct detection by LUX, WMAP and Planck observations, as well as Run 1 results, and (right) in the $m_{\mathrm{DM}}-\sigma_{\mathrm{SI}}$ plane, for a vector mediator assumption.

\section{Searches for diboson resonances}

The searches for narrow resonances decaying to pairs of boosted vector bosons, either $W$ or $Z$, are performed using the decay channels of both bosons decaying hadronically, or one of the $W$ bosons decaying semileptonically (either to an electron or to a muon) and a neutrino [26]. The results performed with Run 1 data by the ATLAS [27] and the CMS experiments $[28,29]$ show a small excess around diboson mass of $1.8 \mathrm{TeV}$, by combining the multiple final states in hadronic and semileptonic decays, which has received large attention, see, for example, Ref. [30].

The main challenge of the analysis is to reconstruct the hadronic decays of highly boosted $W$ or $Z$ bosons and separate them from the background events. Dedicated algorithms are developed using the substructure of boosted jets, the so-called "V tagging" [31, 32]. In order to increase the sensitivity, the events are further categorized according to the so-called $N$-subjettiness [33] output. The analysis is performed using the reconstructed invariant mass distributions of two identified $\mathrm{V}$ jets in comparison with the SM expected backgrounds. No large deviations are found with respect to the expectations and limits are set. The exclusion limits are interpreted in terms of signal models under consideration ( $W^{\prime}$ or bulk Graviton resonances), which are shown in Fig. 12, by combining the hadronic and the semileptonic decay channels. More stringent upper limits on the cross section times branching fraction are set for the considered signal models. 

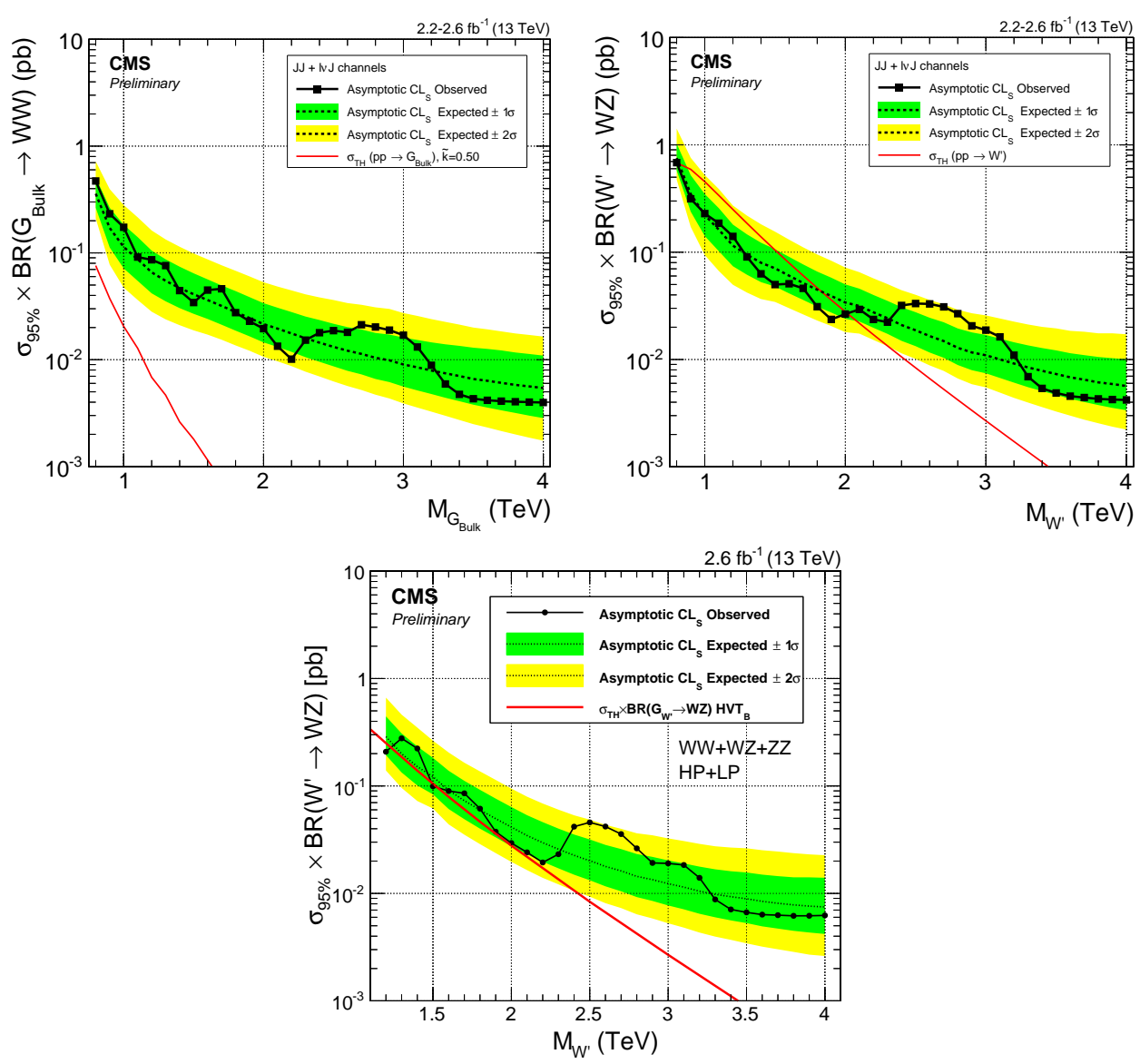

Fig. 12. Observed and expected upper limits from the combination of hadronic and semileptonic decay channels of dibosons (left top) for the bulk graviton $\left(G_{\mathrm{bulk}} \rightarrow\right.$ $W W$ ) mode, (right top) for $W^{\prime} \rightarrow W Z$ decays, and (bottom) combination of the hadronic decays for a $W^{\prime} \rightarrow W Z$ decay mode.

\section{Searches for diphoton resonances}

Two types of theoretical models (ADD [34] and RS [35]) aim to solve the hierarchy problem by suggesting the existence of extra space dimensions (flat or warped) where the gravity is allowed to propagate. Both models predict new spin-2 resonances known as "gravitons", excitations of gravitational fields. The searches for narrow spin-2 resonances that mainly focus on the RS models are performed using the distributions of the diphoton events, corresponding to $2.6 \mathrm{fb}^{-1}$ of collected Run 2 collision data [36]. 
Three different effective couplings $(\tilde{\kappa})$ in the range of $0.01-0.2$ and for the resonance masses $\left(M_{\mathrm{G}}\right)$ in the range of $500 \mathrm{GeV}-4.5 \mathrm{TeV}$ are considered. In order to increase the sensitivity, the events are further categorized in barrel-barrel (EBEB) and barrel-endcap (EBEE) events, where one of the photon objects is required to be reconstructed in the barrel region of the electromagnetic calorimeter. Figure 13 shows the invariant mass distribution and the expected background parametrized as $f\left(m_{\gamma \gamma}\right)=m_{\gamma \gamma}^{a+b} \log m_{\gamma \gamma}$, where $m_{\gamma \gamma}$ is the invariant mass of the diphoton pair.
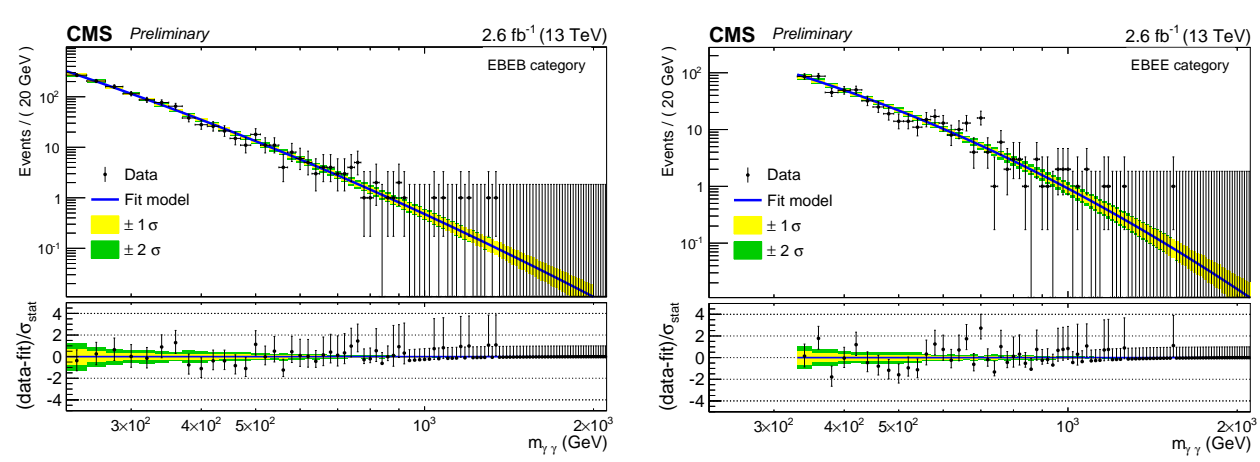

Fig. 13. The invariant mass spectrum of diphoton events, corresponding to $2.6 \mathrm{fb}^{-1}$ Run 2 data according to the categorization of events: (left) in the barrel-barrel (EBEB) and (right) in the barrel-endcap (EBEE) region of the detector. The predicted background event shapes are shown as well.

The analysis is performed as a search for a narrow resonance in the observed diphoton invariant mass spectrum. The results of the observed local $p$-values, which are evaluated with the background-only scenarios for three different effective couplings, 0.01, 0.1 and 0.2, are shown in Fig. 14 (left), respectively, where the largest excess is observed around the mass of $760 \mathrm{GeV}$, with a local significance of 2.6 standard deviations for an effective coupling value of 0.01 , i.e. the narrow width scenario. By taking into account the so-called look elsewhere effect, the significance of this observation is reduced to a global $p$-value of 1.2 standard deviations. The observation of the largest excess is not compatible with a new RS signal present in the collected data, and upper limits are set for corresponding signal models, which are shown in Fig. 14 (right). The Run 2 analysis excludes RS gravitons with masses below $1.35,3.1$ and $3.8 \mathrm{TeV}$ for $\tilde{\kappa}$ of $0.01,0.1$ and 0.2 , respectively. These mass limits are already better than the ones obtained with the $8 \mathrm{TeV}$ collision data [37]. 

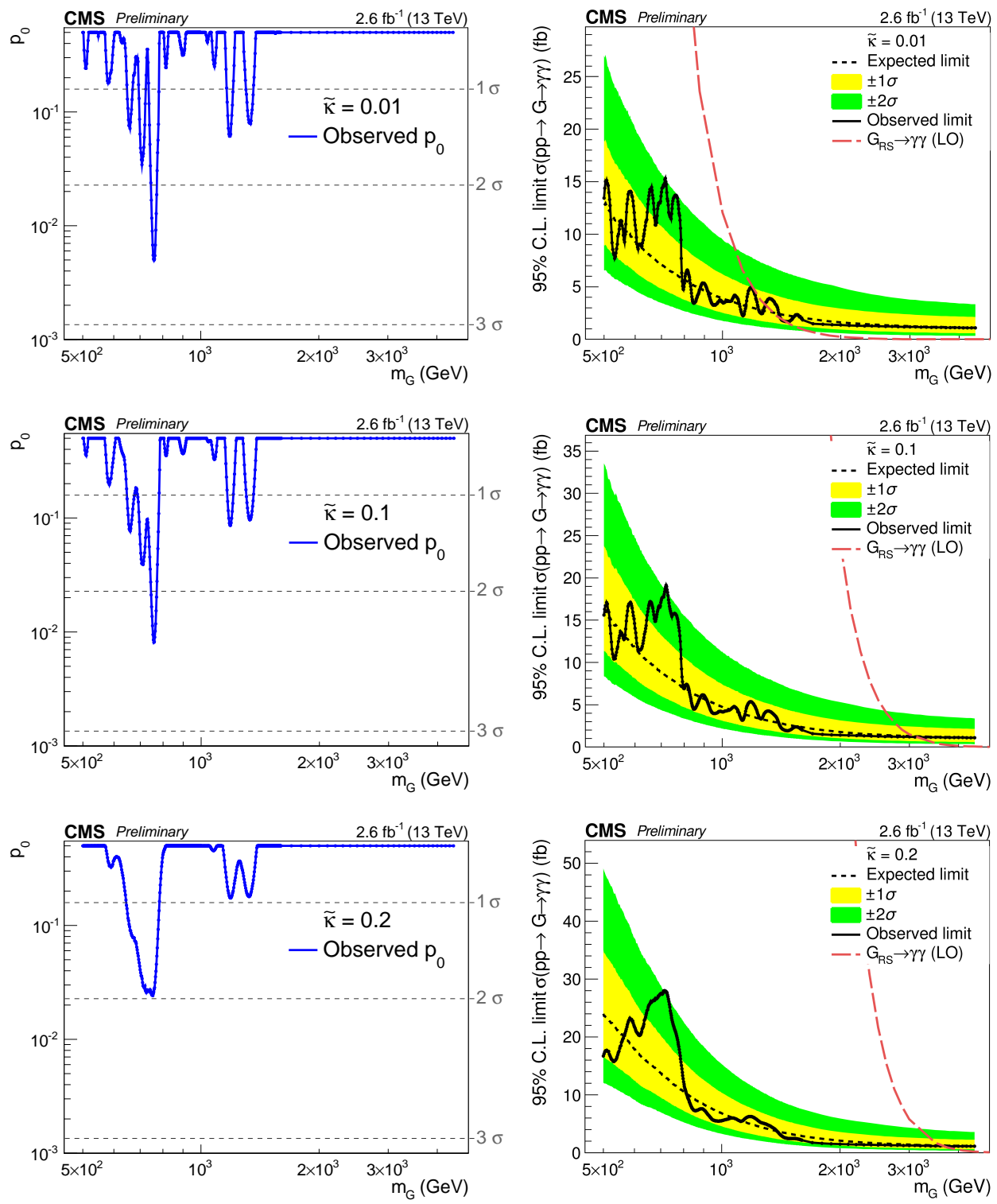

Fig. 14. Left: The background-only local $p$-values. Right: Observed and expected limits at $95 \%$ C.L. Results are shown for three different RS signal width hypotheses, from top to bottom: $\tilde{\kappa}=0.01, \tilde{\kappa}=0.1$, and $\tilde{\kappa}=0.2$, respectively. 
Figure 15 shows comparisons, as well as the combination, of the local $p$-values from Run 1 and Run 2 analyses, assuming the narrow width scenario for the signal model. By combining the two analyses, the observation of the local $p$-value is found to be increased to 3.0 standard deviations for the signal mass value of $750 \mathrm{GeV}$.

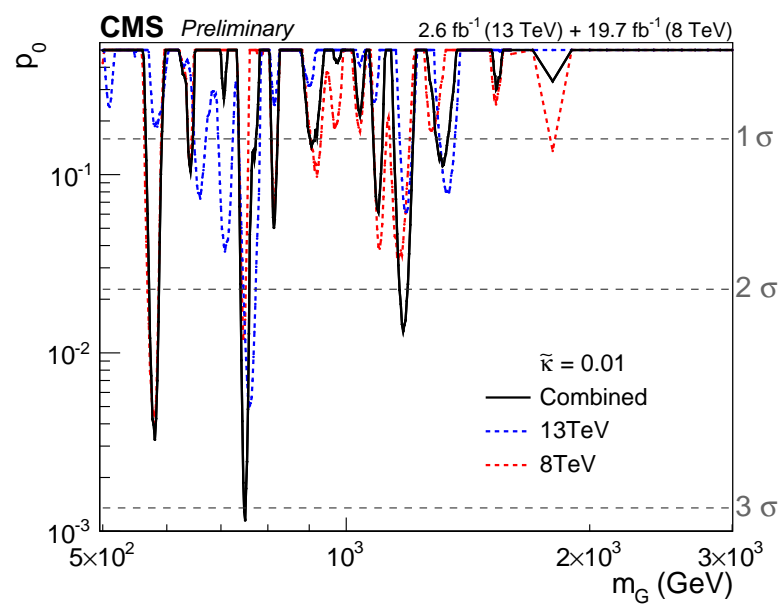

Fig. 15. Observed background-only $p$-values obtained from the combination of Run $1(8 \mathrm{TeV})$ and Run 2 results.

\section{Summary}

Searches for exotic phenomena at the CMS experiment cover various topics. A subset of the first Run 2 analyses results is presented, mostly focusing on the searches for new resonances (spin-1 or spin-2) as well as dark matter and Black Holes. Most of the results obtained with Run 2 searches already extend and surpass the results previously published with Run 1 data. The results of diboson analyses obtained with Run 2 data do not show any significant excess in the invariant mass region where Run 1 analyses reported a light excess. A new excess has been observed in the diphoton mass spectrum, making upcoming LHC proton-proton collision data at $13 \mathrm{TeV}$ center-of-mass energies even more interesting and exciting.

\section{REFERENCES}

[1] S. Chatrchyan et al., Phys. Lett. B 716, 30 (2012).

[2] G. Aad et al., Phys. Lett. B 716, 1 (2012).

[3] S. Chatrchyan et al., JINST 3, S08004 (2008). 
[4] Exotica public results, http://cms-results.web.cern.ch/cms-results/ public-results/publications/EXO/index.html

[5] B2g prelimenary results, http://cms-results.web.cern.ch/cms-results/public-results/ preliminary-results/LHC-Jamboree-2015/B2G.html

[6] V. Khachatryan et al., Phys. Rev. Lett. 116, 071801 (2016).

[7] S. Chatrchyan et al., Phys. Rev. D 87, 114015 (2013).

[8] CMS Collaboration, Searches for Quark Contact Interactions and Extra Spatial Dimensions with Dijet Angular Distributions in Proton-Proton Collisions at 13 TeV, CMS-PAS-EXO-15-009, 2015.

[9] CMS Collaboration, Search for a Narrow Resonance Produced in $13 \mathrm{TeV}$ $p p$ Collisions Decaying to Electron Pair or Muon Pair Final States, CMS-PAS-EXO-15-005, 2015.

[10] V. Khachatryan et al., J. High Energy Phys. 1504, 025 (2015).

[11] S. Başeğmez, A New Method for Mapping Detector Material in situ and a Matrix Element Approach to the Search for Heavy Di-muon Resonances at the LHC, Ph.D. Thesis, Université Catholique de Louvain, 2015.

[12] P. Artoisenet, V. Lemaitre, F. Maltoni, O. Mattelaer, J. High Energy Phys. 1012, 068 (2010).

[13] F. Maltoni, T. Stelzer, J. High Energy Phys. 0302, 027 (2003).

[14] CMS Collaboration, Search for SSM $W^{\prime}$ Production in the Lepton+MET Final State at a Center-of-mass Energy of 13 TeV, CMS-PAS-EXO-15-006, 2015.

[15] CMS Collaboration, Search for Leptonic Decays of $W^{\prime}$ Bosons in $p p$ Collisions at $\sqrt{s}=8 \mathrm{TeV}$, CMS-PAS-EXO-12-060, 2013.

[16] CMS Collaboration, Search for $W^{\prime}$ Boson Resonances Decaying into a Top Quark and a Bottom Quark in the Leptonic Final State at $\sqrt{s}=13 \mathrm{TeV}$, CMS-PAS-B2G-15-004, 2015.

[17] S. Chatrchyan et al., J. High Energy Phys. 1405, 108 (2014).

[18] CMS Collaboration, Search for Black Holes with Early Run 2 Data, CMS-PAS-EXO-15-007, 2015.

[19] S. Chatrchyan et al., J. High Energy Phys. 1307, 178 (2013).

[20] CMS Collaboration, Search for Dark Matter with Jets and Missing Transverse Energy at 13 TeV, CMS-PAS-EXO-15-003, 2015.

[21] D. Abercrombie et al., Dark Matter Benchmark Models for Early LHC Run 2 Searches: Report of the ATLAS/CMS Dark Matter Forum, 2015.

[22] V. Khachatryan et al., Eur. Phys. J. C 75, 235 (2015).

[23] CMS Collaboration, Search for New Physics in the $V$-jet+MET Final State, CMS-PAS-EXO-12-055, 2015.

[24] C.L. Bennett et al., Astrophys. J. 583, 1 (2003).

[25] J. Tauber et al., arXiv:astro-ph/0604069. 
[26] CMS Collaboration, Search for Massive Resonances Decaying into Pairs of Boosted $W$ and $Z$ Bosons at $\sqrt{s}=13$ TeV, CMS-PAS-EXO-15-002, 2015.

[27] G. Aad et al., Eur. Phys. J. C 75, 209 (2015) [Erratum ibid. 75, 370 (2015)].

[28] V. Khachatryan et al., J. High Energy Phys. 1408, 174 (2014).

[29] V. Khachatryan et al., J. High Energy Phys. 1408, 173 (2014).

[30] F. Dias et al., J. High Energy Phys. 1604, 155 (2016).

[31] S.D. Ellis, C.K. Vermilion, J.R. Walsh, Phys. Rev. D 80, 051501 (2009).

[32] S.D. Ellis, C.K. Vermilion, J.R. Walsh, Phys. Rev. D 81, 094023 (2010).

[33] J. Thaler, K. Van Tilburg, J. High Energy Phys. 1103, 015 (2011).

[34] N. Arkani-Hamed, S. Dimopoulos, G.R. Dvali, Phys. Lett. B 429, 263 (1998).

[35] L. Randall, R. Sundrum, Phys. Rev. Lett. 83, 3370 (1999).

[36] CMS Collaboration, Search for New Physics in High Mass Diphoton Events in Proton-Proton Collisions at 13 TeV, CMS-PAS-EXO-15-004, 2015.

[37] CMS Collaboration, Search for High-Mass Diphoton Resonances in $p p$ Collisions at $\sqrt{s}=8 \mathrm{TeV}$ with the CMS Detector, CMS-PAS-EXO-12-045, 2015. 